\title{
Effect of post-pretreatment washing on saccharification and co-fermentation from bagasse pretreated with biocompatible cholinium ionic liquid
}

Kazuaki Ninomiya $^{\mathrm{a}}$, Chiaki Ogino $^{\mathrm{b}}{ }$, Miki Ishizaki $^{\mathrm{b}},{\text { Misa } \text { Yasuda }^{\mathrm{b}} \text {, Nobuaki Shimizu }}^{\mathrm{a}}$, and Kenji Takahashi ${ }^{\mathrm{C}} *$

${ }^{a}$ Institute of Nature and Environmental Technology, Kanazawa University, Kakuma-machi, Kanazawa 920-1192, Japan

${ }^{b}$ Department of Chemical Science and Engineering, Graduate School of Engineering, Kobe University, 1-1 Rokkodaicho, Nada-ku, Kobe 657-8501, Japan

${ }^{c}$ Faculty of Natural System, Institute of Science and Engineering, Kanazawa University, Kakuma-machi, Kanazawa 920-1192, Japan

* Corresponding authors.

Tel.: +8178 803 6193; fax: +81788036193

E-mail address: ochiaki@ port.kobe-u.ac.jp (C. Ogino)

Tel.: +8176234 4828; fax: +8176234 4829

E-mail address: ktkenji@staff.kanazawa-u.ac.jp (K. Takahashi) 


\begin{abstract}
Choline acetate (ChOAc), a cholinium ionic liquid (IL), was used for biomass pretreatment; thereafter, saccharification of the pretreated biomass and glucose/xylose co-fermentation were conducted with different number of post-pretreatment washes (1-5 and 10 washes). Besides saccharification and co-fermentation data, the inhibitory effect of ChOAc on the biocatalyst was compared with that of 1-ethyl-3-methylimidazolium acetate (EmimOAc). ChOAc showed less inhibitory effect on cellulase and yeast activity compared with EmimOAc. The cellulose and hemicellulose saccharification percentages of the IL-pretreated bagasse were approximately $90 \%$ and 60\%, respectively, irrespective of ChOAc and EmimOAc with sufficient post-pretreatment washes. The cellulose and hemicellulose saccharification percentages after 5 washes were $82 \%$ and $59 \%$, respectively, in the case of ChOAc and $51 \%$ and $13 \%$, respectively, in the case of EmimOAc. The overall ethanol yields on the original bagasse basis after saccharification and co-fermentation of the IL-pretreated bagasse when washed 5 times were $54 \%$ and $22 \%$ in the case of ChOAc and EmimOAc, respectively. ChOAc-pretreated bagasse could be saccharified and co-fermented with fewer washes than EmimOAc-pretreated bagasse.
\end{abstract}

\title{
Keywords:
}

Ionic liquid; Pretreatment; Cellulose; Fermentation; Ethanol; Yeast. 


\section{Introduction}

Some types of ionic liquids (ILs; generally defined as organic salts that melt below $100^{\circ} \mathrm{C}$ ) have been demonstrated to dissolve cellulose [1]. Moreover, the reprecipitated cellulose after dissolution in ILs has been shown to have a higher efficiency for enzymatic saccharification, because its crystallinity is significantly decreased [2]. This IL-assisted pretreatment method has been applied to several types of lignocellulosic materials [3]. Moreover, the IL-assisted pretreatment method has been demonstrated to be more effective than the conventional pretreatment method using diluted acid or ammonia [4-7]. In the case of diluted acid pretreatment, most hemicellulose is hydrolyzed and washed out during the pretreatment procedure. On the other hand, hemicellulose can remain in the IL-pretreated biomass, indicating that the IL-pretreated biomass can be suitable for glucose/xylose co-fermentation after enzymatic saccharification [7].

However, the IL-pretreated biomass needs to be washed extensively to remove the residual IL, because even a small amount of residual IL in the pretreated biomass inhibits cellulolytic enzymes and fermentative microbes in the subsequent saccharification and fermentation steps [8,9]. These inhibitory effects are much more marked, particularly in the case of high solid loading of IL-pretreated biomass to the saccharification and fermentation processes. Extensive washing of the pretreated biomass results in large amounts of diluted IL aqueous solution, which leads to high costs for both concentrating the IL from its diluted aqueous solution by evaporation and treating the resultant wastewater.

Completely bio-derived cholinium ILs have been reported, which contain either cholinium cations combined with amino acid-based anions [10] or carboxylic 
acid-based anions [11]. Moreover, it has been demonstrated that these cholinium ILs could be used for biomass pretreatment to enhance enzymatic saccharification of lignocellulosic biomass [12-18], similar to conventional imidazolium ILs. Furthermore, our previous study demonstrated that cholinium ILs were less inhibitory to cellulase enzymes and fermentative microorganisms than imidazolium ILs [13,18]. For imidazolium ILs, there are a few studies reporting the effect of the number of post-pretreatment washes on the resulting inhibition of enzymatic saccharification of the IL-pretreated biomass $[19,20]$. However, to the best of our knowledge, there are no studies reporting the effect of the number of post-pretreatment washes on both saccharification and co-fermentation of the cholinium IL-pretreated biomass.

Therefore, the present study investigated the effect of the number of post-pretreatment washes on the saccharification and subsequent co-fermentation of the cholinium IL-pretreated biomass. Choline acetate (ChOAc) was used as the biocompatible cholinium IL. The data for enzymatic saccharification and subsequent microbial ethanol co-fermentation were compared with data obtained using 1-ethyl-3-methylimidazolium acetate (EmimOAc), the conventional imidazolium IL, frequently employed for biomass pretreatment.

\section{Materials and Methods}

\subsection{Biomass, IL, cellulase, and yeast cells}

Bagasse powder with a particle size of $>200 \mu \mathrm{m}$ was purchased from Toyota Motor Corporation (Miyoshi, Japan). ChOAc was prepared using a one-pot neutralization method with minor modifications [21]. EmimOAc was purchased from Kanto Chemical Co., Inc. (Tokyo, Japan). Commercial cellulase (Cellic® CTec2), a complex blend of 
cellulase, hemicellulose, and $\beta$-glucosidase, was obtained from Novozymes Japan Ltd (Chiba, Japan). Filter paper unit (FPU) of the cellulase was determined by the NREL method [22]. All other chemicals were from commercial sources and of reagent grade. Saccharomyces cerevisiae YPH499XU, which co-expresses xylose reductase from Pichia stipites, xylitol dehydrogenase from $P$. stipites and xylulokinase from $S$. cerevisiae [23], was used throughout the study as the test yeast strain assimilating both glucose and xylose.

\subsection{Assay of yeast growth inhibition by ILs}

For evaluating the inhibitory effects of IL to yeast, the culture was conducted in a 5-mL test tube containing autoclaved YPD medium $(10 \mathrm{~g} / \mathrm{L}$ of yeast extract, $20 \mathrm{~g} / \mathrm{L}$ of Bacto-peptone, and 20 g/L of glucose; Nacalai Tesque, Kyoto, Japan) supplemented with the IL (EmimOAc or ChOAc) at final concentrations of $0.3 \%-5 \%(\mathrm{w} / \mathrm{w})$ The yeast cells were precultured aerobically at $30^{\circ} \mathrm{C}$ in the test tube containing $5 \mathrm{~mL}$ of YPD medium free from IL. The precultured broth was transferred into a test tube at an initial optical density of 0.1 at a wavelength of $\underline{660 \mathrm{~nm}\left(\mathrm{OD}_{660}\right)}$. The test tube was incubated at $30^{\circ} \mathrm{C}$ for $48 \mathrm{~h}$ at $48 \mathrm{rpm}$ using a shaking incubator (Compact Rocking Incubator TVS062CA, Advantec Toyo Kaisha, Ltd., Tokyo, Japan) with the $\mathrm{OD}_{660}$ value being monitored at 10-min interval. The response to the IL was evaluated based on the relative growth, which was defined as the percentage of the $\mathrm{OD}_{660}$ at $48 \mathrm{~h}$ in the presence of the IL relative to the $\mathrm{OD}_{660}$ at $48 \mathrm{~h}$ of a control culture free from the IL. The median effective concentration based on yeast growth $\left(\mathrm{yEC}_{50}\right)$ was determined as the $\mathrm{IL}$ concentration at which the relative growth was reduced to $50 \%$. 


\subsection{IL-assisted pretreatment and washing of biomass}

For biomass pretreatment, $0.5 \mathrm{~g}$ of bagasse powder was added to a $15-\mathrm{mL}$ polypropylene tube (Corning Inc., New York, United States) containing $5 \mathrm{~g}$ of IL (EmimOAc or $\mathrm{ChOAc)}$. After vortexing, the biomass/IL mixture in the tube was sonicated for $30 \mathrm{~min}$ at $28 \mathrm{kHz}$ and an emission power of $25 \mathrm{~W}$ using an ultrasonic processor (UD-211 with sonotrode TP-040, Tomy Seiko Co., Ltd. Tokyo, Japan) in a water bath maintained at $25^{\circ} \mathrm{C}$, so as to enhance the IL assisted pretreatment [15].

For washing out the IL from the pretreated biomass, $45 \mathrm{~mL}$ of deionized water was added to the bagasse/IL mixture in a $50-\mathrm{mL}$ tube, which resulted in the precipitation of the biomass. After stirring, the 50 -mL tube was centrifuged $(8,000 \times g)$ for $10 \mathrm{~min}$ at $25^{\circ} \mathrm{C}$, and the supernatant was removed. The washing procedure was repeated $1-5$ and 10 times. The precipitated wet bagasse was used for subsequent enzymatic saccharification.

\subsection{Saccharification and co-fermentation at high loading}

Saccharification was performed in a 50-mL polypropylene tube (Corning Inc.) containing the wet pretreated biomass (water content was adjusted to $90 \%$, which resulted in $10 \%$ solid loading) and CTec2 at a concentration of $20 \mathrm{FPU} / \mathrm{g}$ pretreated biomass. The tube was set in a heat block (Thermo Block Rotator SN-06BN; Nissinrika Co., Tokyo, Japan) and axially rotated at $35 \mathrm{rpm}$ under a controlled temperature of $\underline{50^{\circ} \mathrm{C}}$. During the enzymatic reaction, liquefied samples were collected after 3, 24, 48, and 72 $\mathrm{h}$ and then heated at $90^{\circ} \mathrm{C}$ for 5 min to inactivate the enzyme. After centrifugation of the heated samples at $21,500 \times g$ for $1 \mathrm{~min}$, the supernatant was subjected to glucose and xylose measurement. Cellulose saccharification was evaluated as the percentage of 
cellulose hydrolyzed into glucose compared with cellulose in the original bagasse. Similarly, hemicellulose saccharification was evaluated as the percentage of hemicellulose hydrolyzed into xylose compared with hemicellulose in the original bagasse.

After enzymatic saccharification for $72 \mathrm{~h}$, the 50 -mL tube was centrifuged $(8,000 \times$ $g$ ) for $10 \mathrm{~min}$ at $25^{\circ} \mathrm{C}$, and the supernatant was filtered using a $0.45-\mu \mathrm{m}$ filter to obtain a sterile sugar solution for subsequent fermentation. As an inoculum for fermentation, the yeast was aerobically cultured for $72 \mathrm{~h}$ at $30^{\circ} \mathrm{C}$ and $150 \mathrm{rpm}$ in $5 \mathrm{~mL}$ of YPD medium. The yeast cells were harvested by centrifugation at 3,000 rpm for $5 \mathrm{~min}$ and washed twice with distilled water. Fermentation was then performed in a $50-\mathrm{mL}$ polypropylene tube closed with a silicon plug (AS ONE, Osaka, Japan), into which a hole was bored using a disposable needle (Terumo Corp., Tokyo, Japan). The obtained sugar solution, water, and yeast suspension were added to a $50-\mathrm{mL}$ test tube to obtain the working volume of $10 \mathrm{~mL}$ and a final $\mathrm{OD}_{600}$ of 20 (approximately equivalent to $100 \mathrm{~g}$-wet cells/L). Nutrients such as yeast extract and peptone were not added to the fermentation mixture. The tube was set in a reciprocating shaker maintained at $30^{\circ} \mathrm{C}$. During fermentation, samples were collected after 3, 24, and $48 \mathrm{~h}$. After centrifugation of the samples at $21,500 \times g$ for $1 \mathrm{~min}$, the supernatant was subjected to glucose, xylose, and ethanol measurement. The overall ethanol yield on a bagasse basis was evaluated as the percentage of ethanol compared with the ethanol theoretically converted from carbohydrate in the original bagasse. Similarly, the ethanol yield on a sugar basis was evaluated as the percentage of ethanol compared with the ethanol theoretically converted from glucose and xylose obtained by saccharification. 


\subsection{Analysis of biomass composition}

The cellulose, hemicellulose, and lignin contents of the original bagasse were determined according to the NREL method [24] with minor modifications. In brief, 0.1 $\mathrm{g}$ of the sample was mixed with $2 \mathrm{~mL}$ of a $72 \%(v / v) \mathrm{H}_{2} \mathrm{SO}_{4}$ aqueous solution for $2 \mathrm{~h}$ at room temperature. The mixture was transferred to a 200-mL Erlenmeyer flask, diluted with $75 \mathrm{~mL}$ of water, and autoclaved at $121^{\circ} \mathrm{C}$ for $15 \mathrm{~min}$. The acid-diluted hydrolysate was filtered, after which the amount of acid-insoluble lignin including ash was gravimetrically determined by measuring the residue on the filter after drying at $100^{\circ} \mathrm{C}$ for $12 \mathrm{~h}$. The amount of acid-soluble lignin was determined from the ultraviolet (UV) absorbance of the filtrate at $205 \mathrm{~nm}$ and an absorption coefficient of $110 \mathrm{~L} / \mathrm{g} \mathrm{cm}$. The sum of the acid-insoluble and acid-soluble lignin was regarded as the total lignin amount. The amount of glucose and xylose in the hydrolysate was determined using assay kits described below. The amount of cellulose and hemicellulose was calculated from the glucose and xylose content multiplied by anhydro correction factors of 162/180 and 132/150, respectively. The composition was as follows: cellulose $39 \%$, hemicellulose $28 \%$, lignin andn ash $33 \%$.

\subsection{Analysis of glucose, xylose, and ethanol}

Glucose and xylose concentration was measured using glucose and xylose kits, respectively, as per the manufacturer's instruction (Glucose C-II test Wako, Wako Pure Chemical Industries, Ltd., Osaka, Japan; and D-Xylose Assay Kit, Megazyme International Ireland, Wicklow, Ireland). Ethanol concentration was determined using a gas chromatography (GC) system (model GC-2010 Plus, Shimadzu, Kyoto, Japan) equipped with a flame ionization detector (FID) and a DB-FFAP capillary column $(0.25$ 
mm ID, Agilent Technologies, Santa Clara, California, United States). The column temperature was initially maintained at $40^{\circ} \mathrm{C}$ for $1 \mathrm{~min}$ and then increased to $170^{\circ} \mathrm{C}$ at $10^{\circ} \mathrm{C}$ per min, then again to $230^{\circ} \mathrm{C}$ at $15^{\circ} \mathrm{C}$ per min, and finally maintained at this temperature for $5 \mathrm{~min}$.

\section{Results and Discussion}

\subsection{Pretreatment capability of ChOAc or EmimOAc}

To examine the pretreatment capability of ChOAc or EmimOAc for subsequent enzymatic hydrolysis of the lignocellulosic biomass, the bagasse powder was pretreated with each IL and then hydrolyzed by Ctec 2 cellulase for $72 \mathrm{~h}$. The pretreated bagasse was washed 10 times to sufficiently remove the IL before saccharification to eliminate any inhibitory effect of the IL on the cellulolytic enzymes. Table 1 lists the saccharification percentage of ChOAc- or EmimOAc-pretreated bagasse at $72 \mathrm{~h}$. With ChOAc pretreatment, the cellulose and hemicellulose saccharification percentages were $93 \%$ and $65 \%$, respectively, at $72 \mathrm{~h}$. These values are approximately the same as those using EmimOAc (Table 1). In contrast, without any IL pretreatment, the cellulose and hemicellulose saccharification percentages were $24 \%$ and $9 \%$, respectively, of the theoretical maximum at $72 \mathrm{~h}$ (Table 1). These results indicate that ChOAc had sufficient pretreatment capability for lignocellulosic materials, which was almost at the same level as EmimOAc under the examined pretreatment condition.

EmimOAc has frequently been considered as the most effective IL for cellulose dissolution and pretreatment for enzymatic hydrolysis [3]. In general, anions act as hydrogen bond acceptors that interact with the hydroxyl group of cellulose, thereby weakening the crystalline structure of cellulose [25]. Among the anions of imidazolium 
ILs, the acetate anion has been reported to exhibit the highest hydrogen bond basicity ( $\beta$ ) and capability of reducing cellulose crystallinity [3]. This is why pretreatment with ChOAc also enhanced the saccharification of cellulose and hemicellulose to the same degree as pretreatment with EmimOAc (Table 1).

\subsection{Inhibitory effect of ChOAc or EmimOAc on cellulolytic enzymes and yeast cells}

Our previous study [18] reported the inhibitory effect of ChOAc or EmimOAc on cellulase enzymes, where enzymatic saccharification of phosphoric acid swollen cellulose (PASC) was conducted for $24 \mathrm{~h}$ using Ctec 2 cellulase in the presence of the prescribed concentration of each IL. The median effective concentration $\left(\mathrm{EC}_{50}\right)$ values based on cellulase activity $\left(\mathrm{cEC}_{50}\right)$, determined as the IL concentration at which the relative cellulase activity was reduced to $50 \%$, were $32 \mathrm{wt} \%$ and $16 \mathrm{wt} \%$ for ChOAc and EmimOAc, respectively, from the dose-response relationships between the relative enzyme activity and IL concentration. Here, to examine the inhibitory effect of ChOAc or EmimOAc on yeast cells, $S$. cerevisiae YPH499XU was cultured for $48 \mathrm{~h}$ in the presence of the prescribed concentration of each IL. In the case of ChOAc, the relative growth was over $80 \%$ of the control under the examined concentration (Fig. 1). The $\mathrm{EC}_{50}$ values based on yeast growth $\left(\mathrm{yEC}_{50}\right)$ were determined from the dose-response relationships between relative yeast growth and IL concentration. As listed in Table 1, the $\mathrm{yEC}_{50}$ value could not be determined for ChOAc under the examined concentrations and was $0.6 \mathrm{wt} \%$ for EmimOAc. These results indicate that ChOAc shows less inhibitory effect on cellulase and yeast activity compared with EmimOAc under the examined conditions.

Enzymes in IL aqueous solution generally lose their activity because of higher salt 
concentrations and ionic strength. Microbes also lose their viability due to enzyme inhibition and cell membrane damage by IL. Thus, a more biocompatible IL was needed to perform saccharification of the IL-pretreated biomass with fewer post-pretreatment washes. The inhibitory effects of ChOAc on cellulase and yeast cells were significantly lower than those of EmimOAc (Table 1), because the cholinium cation is reported to be more intrinsically biocompatible than the imidazolium cation $[13,18,26,27]$. On the other hand, in addition to the biocompatible IL-like ChOAc, IL-tolerant cellulase $[8,28,29]$ and IL-tolerant fermentative microorganisms [30,31] are also effective in further reducing the number of post-pretreatment washes and the resultant wastewater of the diluted IL aqueous solution.

\subsection{Saccharification of ChOAc- or EmimOA-pretreated biomass with different number of washes}

To examine the saccharification efficiency of ChOAc- or EmimOAc-pretreated biomass with different number of post-pretreatment washes, the pretreated bagasse powders were washed 1-5 and 10 times and then subjected to enzymatic saccharification using Ctec 2 for $72 \mathrm{~h}$. The recovered bagasse from the pretreatment was around $90 \%$ of the original, irrespective of the washing times and ionic liquids. According to the time courses of glucose and xylose concentration during the enzymatic reaction of the pretreated bagasse, the concentrations reached the saturated values up to $72 \mathrm{~h}$ irrespective of the ILs and number of washes (data not shown). Figure 2 shows the cellulose and hemicellulose saccharification percentages obtained at $72 \mathrm{~h}$ of saccharification of ChOAc- or EmimOAc-pretreated bagasse with different number of washes. With 10 post-pretreatment washes, the cellulose and hemicellulose percentages 
were approximately $90 \%$ and $65 \%$, respectively, irrespective of the ILs, which corresponded to approximately $39 \mathrm{~g} / \mathrm{L}$ and $19 \mathrm{~g} / \mathrm{L}$, respectively. The saccharification percentages decreased as the number of washes decreased for both ChOAc and EmimOAc. The saccharification percentages in the case of ChOAc were higher than those in the case of EmimOAc within the range of 1-5 washes. The cellulose and hemicellulose saccharification percentages for 5 washes were $82 \%$ and $59 \%$, respectively, in the case of ChOAc and $51 \%$ and $13 \%$, respectively, in the case of EmimOAc. Even with 4 washes, the cellulose and hemicellulose saccharification percentages for ChOAc reached $75 \%$ and $61 \%$, respectively, although the cellulose and hemicellulose saccharification percentages were just $32 \%$ and $11 \%$, respectively, in the case of EmimOAc. These results indicate that ChOAc-pretreated bagasse could be saccharified with fewer washes than EmimOAc-pretreated bagasse.

High solid loading (at over $15 \% \mathrm{w} / \mathrm{w}$ ) in the pretreatment step [32] and saccharification step [33] can reduce the amount of wastewater during the conversion process of lignocellulosic material to the fermentation product, because high solid loading can reduce the reaction scale and therefore enhance the concentration of sugars and fermentation product, resulting in the reduction of the capital and running cost. With regard to high solid loading in the IL-assisted pretreatment step, $15 \%-50 \%$ of solid loading has been reported [14,20,34-36]. When these IL-pretreated biomasses are washed and then subjected to subsequent high-solid saccharification, the concentration of the IL carried over into the subsequent enzymatic reaction cannot be neglected because the water content for the saccharification system is low. In these situations, biocompatibility of the IL and IL tolerance of the biocatalyst are very critical. ChOAc, which was demonstrated to have pretreatment capability and biocompatibility, is also 
advantageous for pretreatment and subsequent saccharification at high solid loading.

\subsection{Co-fermentation of IL-pretreated biomass with different number of washes}

To examine the co-fermentation efficiency of mixed sugars obtained from the saccharification of ChOAc- or EmimOAc-pretreated biomass with different number of post-pretreatment washes, the pretreated bagasse powders were washed 1-5 and 10 times. They were then subjected to enzymatic saccharification using Ctec 2 for $72 \mathrm{~h}$, followed by ethanol fermentation using the xylose assimilating recombinant yeast YPH499XU for another $48 \mathrm{~h}$. Figure 3 shows the time courses of glucose, xylose, and ethanol concentration during fermentation. Although the initial sugar concentration was different depending on the ILs and post-pretreatment washes, glucose was consumed by $24 \mathrm{~h}$. Xylose concentration decreased gradually, but did not reduce to zero within $48 \mathrm{~h}$ of fermentation. Ethanol concentration reached the saturation values by $48 \mathrm{~h}$ irrespective of the ILs and number of washes. With 10 post-pretreatment washes, ethanol concentration reached approximately $15 \mathrm{~g} / \mathrm{L}$ irrespective of ChOAc and EmimOAc. Conversely, with 2-5 post-pretreatment washes, the profiles of ethanol concentration were significantly deteriorated in the case of EmimOAc compared with those in the case of ChOAc.

Figure 4A shows the overall ethanol yield on the original bagasse basis obtained at $48 \mathrm{~h}$ of co-fermentation of mixed sugars generated by the saccharification of ChOAc- or EmimOAc-pretreated bagasse with different number of washes. With 10 post-pretreatment washes, the overall ethanol yield was approximately $40 \%$ irrespective

of the ILs. The overall yield decreased as the number of washes decreased from 5 to 1 for both ChOAc and EmimOAc. The overall ethanol yield in the case of ChOAc was 
quite higher than that in the case of EmimOAc with 1-5 washes. The overall ethanol yields with 5 washes were $54 \%$ and $22 \%$ in the case of ChOAc and EmimOAc, respectively. These results indicate that compared with EmimOAc-pretreated bagasse, ChOAc-pretreated bagasse could be saccharified and co-fermented with fewer washes.

Figure 4B shows the ethanol yield on a sugar basis obtained at $48 \mathrm{~h}$ of co-fermentation of mixed sugars generated by the saccharification of ChOAc- or EmimOAc-pretreated bagasse with different number of washes. The ethanol yield fluctuated within the range of $60 \%-90 \%$ under the examined conditions, although the reason of these fluctuation was unclear. And there was no clear difference in the yield between ChOAc and EmimOAc with different number of washes. These results were not consistent with the $\mathrm{yEC}_{50}$ values in Table 1, which indicated that EmimOAc showed a relatively larger inhibitory effect on the yeast YPH499XU activity compared with ChOAc. This inconsistency may be attributed to the inoculum size being larger for ethanol fermentation (initial $\mathrm{OD}_{600}=20$ ) compared with that for the growth inhibition assay (initial $\mathrm{OD}_{600}=0.1$ ). Therefore, in the present study, the ILs mainly inhibited cellulase activity during the saccharification and subsequent co-fermentation of IL-pretreated biomass with less number of post-pretreatment washes. Moreover, these results clarified that hemicellulose saccharification was a main bottleneck to further enhance the ethanol yield on bagasse basis, since the value was about $65 \%$ in the present study (Fig. 2B).

The present study, for the first time, compared a cholinium IL (ChOAc) and imidazolium IL (EmimOAc) systematically from the viewpoint of the pretreatment capability of biomass (Table 1), inhibitory effects on cellulase and yeast (Fig. 1 and Table 1), and saccharification (Fig. 2) and subsequent co-fermentation (Fig. 3). It was 
revealed that ChOAc-pretreated bagasse could be saccharified and co-fermented with fewer washes than EmimOAc-pretreated bagasse (Fig. 4). This was because ChOAc showed almost the same capability of biomass pretreatment for saccharification (Table 1), with less inhibitory effects on cellulase and yeast (Table 1). We therefore suggest that $\mathrm{ChOAc}$ is a more favorable IL for biomass pretreatment prior to saccharification and fermentation, because it can reduce the number of post-pretreatment washes and the resultant wastewater of the diluted IL aqueous solution.

\section{Conclusions}

The present study compared ChOAc and EmimOAc with regard to the pretreatment capability of the biomass, inhibitory effects on cellulase and yeast, and saccharification and subsequent co-fermentation of pretreated bagasse. ChOAc showed almost the same capability of pretreatment for saccharification and less inhibitory effects on cellulase and yeast. As a result, ChOAc-pretreated bagasse could be saccharified and co-fermented with fewer washes than EmimOAc-pretreated bagasse. This could reduce the wastewater of the diluted IL aqueous solution.

\section{Acknowledgments}

This study was supported in part by the Advanced Low Carbon Technology Research and Development Program (ALCA) (Grant number 2100040), the Center of Innovation Science and Technology based Radical Innovation and Entrepreneurship Program (COI STREAM), and the Cross-ministerial Strategic Innovation Promotion Program (SIP), all from the Japan Science and Technology Agency. 


\section{References}

[1] R.P. Swatloski, S.K. Spear, J.D. Holbrey, R.D. Rogers, Dissolution of cellose with ionic liquids, J. Am. Chem. Soc. 124 (2002) 4974-4975.

[2] A.P. Dadi, S. Varanasi, C.A. Schall, Enhancement of cellulose saccharification kinetics using an ionic liquid pretreatment step, Biotechnol. Bioeng. 95 (2006) 904-910.

[3] A. Brandt, J. Gräsvik, J.P. Hallett, T. Welton, Deconstruction of lignocellulosic biomass with ionic liquids, Green Chem. 15 (2013) 550-583.

[4] C. Li, B. Knierim, C. Manisseri, R. Arora, H.V. Scheller, M. Auer, K.P. Vogel, B.A. Simmons, S. Singh, Comparison of dilute acid and ionic liquid pretreatment of switchgrass: Biomass recalcitrance, delignification and enzymatic saccharification, Bioresour. Technol. 101 (2010) 4900-4906.

[5] C. Li, G. Cheng, V. Balan, M.S. Kent, M. Ong, S.P.S. Chundawat, L.C. Sousa, Y.B. Melnichenko, B.E. Dale, B.A. Simmons, S. Singh, Influence of physico-chemical changes on enzymatic digestibility of ionic liquid and AFEX pretreated corn stover, Bioresour. Technol. 102 (2011) 6928-6936.

[6] C. Li, L. Sun, B.A. Simmons, S. Seema, Comparing the recalcitrance of eucalyptus, pine, and switchgrass using ionic liquid and dilute acid pretreatments, Bioenergy Res. 6 (2013) 14-23.

[7] N. Uppugundla, L. da Costa Sousa, S.P. Chundawat, X. Yu, B. Simmons, S. Singh, X. Gao, R. Kumar, C.E Wyman, B.E. Dale, V. Balan, A comparative study of ethanol production using dilute acid, ionic liquid and AFEX pretreated corn stover, Biotechnol. Biofuels. 7 (2014) 72.

[8] S. Datta, B. Holmes, J.I. Park, Z. Chen, D.C. Dibble, M. Hadi, H.W. Blanch, 
B.A. Simmons, R. Sapra, Ionic liquid tolerant hyperthermophilic cellulases for biomass pretreatment and hydrolysis, Green Chem. 12 (2010) 338-345.

[9] M. Ouellet, S. Datta, D.C. Dibble, P.R. Tamrakar, P.I. Benke, C. Li, S. Singh, K.L. Sale, P.D. Adams, J.D. Keasling, B.A. Simmons, B.M. Holmes, A. Mukhopadhyay, Impact of ionic liquid pretreated plant biomass on Saccharomyces cerevisiae growth and biofuel production, Green Chem. 13 (2011) 2743-2749.

[10] S. Hu, T. Jiang, Z. Zhang, A. Zhu, B. Han, J. Song, Y. Xie, W. Li, Functional ionic liquid from biorenewable materials: synthesis and application as a catalyst in direct aldol reactions, Tetrahedron Lett. 48 (2007) 5613-5617.

[11] Y. Fukaya, Y. Iizuka, K. Sekikawa, H. Ohno, Bio ionic liquids: room temperature ionic liquids composed wholly of biomaterials, Green Chem. 9 (2007) $1155-1157$.

[12] Q.P. Liu, X.D. Hou, N. Li, M.H. Zong, Ionic liquids from renewable biomaterials: synthesis, characterization and application in the pretreatment of biomass, Green Chem. 14 (2012) 304-307.

[13] K. Ninomiya, T. Yamauchi, M. Kobayashi, O. Chiaki, N. Shimizu, K. Takahashi, Cholinium carboxylate ionic liquids for pretreatment of lignocellulosic materials to enhance subsequent enzymatic saccharification, Biochem. Eng. J. 71 (2013) $25-29$.

[14] K. Ninomiya, H. Soda, C. Ogino, K. Takahashi, N. Shimizu, Effect of ionic liquid weight ratio on pretreatment of bamboo powder prior to enzymatic saccharification, Bioresour. Technol. 128 (2013) 188-192.

[15] K. Ninomiya, A. Ohta, S. Omote, C. Ogino, K. Takahashi, N. Shimizu, 
Combined use of completely bio-derived cholinium ionic liquids and ultrasound irradiation for the pretreatment of lignocellulosic material to enhance enzymatic saccharification, Chem. Eng. J. 215-216 (2013) 811-818.

[16] K. Ninomiya, T. Yamauchi, C. Ogino, N. Shimizu, K. Takahashi, Microwave pretreatment of lignocellulosic material in cholinium ionic liquid for efficient enzymatic saccharification, Biochem Eng. J. 90 (2014) 90-95.

[17] K. Ninomiya, K. Inoue, Y. Aomori, A. Ohnishi, C. Ogino, N. Shimizu, K. Takahashi, Characterization of fractionated biomass component and recovered ionic liquid during cholinium ionic liquid-assisted pretreatment process with its recycle, Chem Eng. J. 259 (2015) 323-329.

[18] K. Ninomiya, A. Kohori, M. Tatsumi, K. Osawa, T. Endo, R. Kakuchi, C. Ogino, N. Shimizu, K. Takahashi, Ionic liquid/ultrasound pretreatment and in situ enzymatic saccharification of bagasse using biocompatible cholinium ionic liquid, Bioresour. Technol. 176 (2015) 169-174.

[19] Q. Li, Y.-C. He, M. Xian, G. Jun, X. Xu, J.-M. Yang, L.Z. Li, Improving enzymatic hydrolysis of wheat straw using ionic liquid 1-ethyl-3-methyl imidazolium diethyl phosphate pretreatment, Bioresour. Technol. 100 (2009) $3570-3575$.

[20] C. Li, D. Tanjore, W. He, J. Wong, J.L. Gardner, K.L. Sale, B.A. Simmons, S. Singh, Scale-up and evaluation of high solid ionic liquid pretreatment and enzymatic hydrolysis of switchgrass, Biotechnol. Biofuels. 6 (2013) 154.

[21] Y. Yu, X. Lu, Q. Zhou, K. Dong, H. Yao, S. Zhang, Biodegradable naphthenic acid ionic liquids: synthesis, characterization, and quantitative structure-biodegradation relationship, Chemistry. 14 (2008) 11174-11782. 
[22] B. Adney, J. Baker, Measurement of cellulase activities, Technical Report, NREL/TP-510-42628, National Renewable Energy Laboratory, Golden, CO.

[23] T. Hasunuma, K.M. Sung, T. Sanda, K. Yoshimura, F. Matsuda, A. Kondo, Efficient fermentation of xylose to ethanol at high formic acid concentrations by metabolically engineered Saccharomyces cerevisiae, Appl. Microbiol. Biotechnol. 90 (2011) 997-1004.

[24] A. Sluiter, B. Hames, R. Ruiz, C. Scarlata, J. Sluiter, D. Templeton, D. Crocker, Determination of structural carbohydrates and lignin in biomass determination of structural carbohydrates and lignin in biomass, Technical Report, NREL/TP-510-42618, National Renewable Energy Laboratory, Golden, CO.

[25] R.C. Remsing, R.P. Swatloski, R.D. Rogers, G. Moyna, Mechanism of cellulose dissolution in the ionic liquid 1- $n$-butyl-3-methylimidazolium chloride: a ${ }^{13} \mathrm{C}$ and ${ }^{35 / 37} \mathrm{Cl}$ NMR relaxation study on model systems, Chem. Commun. (2006) $1271-1273$.

[26] M. Petkovic, J.L. Ferguson, H.N. Gunaratne, R. Ferreira, M.C. Leitao, K.R. Seddon, L.P.N. Rebelo, C. Silva Pereira, Novel biocompatible cholinium-based ionic liquids - Toxicity and biodegradability, Green Chem. 12 (2010) 643-649.

[27] X.D. Hou, Q.P. Liu, T.J. Smith, N. Li, M.H. Zong, Evaluation of toxicity and biodegradability of cholinium amino acids ionic liquids, PLoS One. 8 (2013) e59145.

[28] J.M. Gladden, J.I. Park, J. Bergmann, V. Reyes-Ortiz, P. D’haeseleer, B.F. Quirino, K.L. Sale, B.A. Simmons, S.W. Singer, Discovery and characterization of ionic liquid-tolerant thermophilic cellulases from a swithchgrass-adapted microbial community, Biotechnol. Biofuels. 7 (2014) 15. 
[29] J. Xu, X. Wang, L. Hu, J. Xia, Z. Wu, N. Xu, B. Dai, B. Wu, A novel ionic liquid-tolerant Fusarium oxysporum BN secreting ionic liquid-stable cellulase: Consolidated bioprocessing of pretreated lignocellulose containing residual ionic liquid, Bioresour. Technol. 181 (2015) 18-25.

[30] I.R. Sitepu, S. Shi, B.A. Simmons, S.W. Singer, K. Boundy-Mills, C.W. Simmons, Yeast tolerance to the ionic liquid 1-ethyl-3-methylimidazolium acetate, FEMS Yeast Res. 14 (2014) 1286-1294.

[31] T.L. Ruegg, E.-M. Kim, B.A Simmons, J.D. Keasling, S.W. Singer, T.S. Lee, M.P. Thelen, An auto-inducible mechanism for ionic liquid resistance in microbial biofuel production, Nat. Commun. 5 (2014) 3490.

[32] A.A. Modenbach, S.E. Nokes, The use of high-solids loadings in biomass pretreatment-a review, Biotechnol. Bioeng. 109 (2012) 1430-1442.

[33] T. Fujii, K. Murakami, T. Endo, S. Fujimoto, T. Minowa, A. Matsushika, S. Yano, S. Sawayama, Bench-scale bioethanol production from eucalyptus by high solid saccharification and glucose/xylose fermentation method, Bioprocess Biosyst. Eng. 37 (2014) 749-754.

[34] H. Wu, M. Mora-Pale, J. Miao, T.V Doherty, R.J. Linhardt, J.S. Dordick, Facile pretreatment of lignocellulosic biomass at high loadings in room temperature ionic liquids, Biotechnol. Bioeng. 108 (2011) 2865-2875.

[35] A.G. Cruz, C. Scullin, C. Mu, G. Cheng, V. Stavila, P. Varanasi, D. Xu, J. Mentel, Y.D. Chuang, B.A. Simmons, S. Singh, Impact of high biomass loading on ionic liquid pretreatment, Biotechnol. Biofuels. 6 (2013) 52.

[36] A.S.A. da Silva, R.S.S. Teixeira, T. Endo, E.P.S. Bon, S.H. Lee, Continuous pretreatment of sugarcane bagasse at high loading in an ionic liquid using a 
twin-screw extruder, Green Chem. 15 (2013) 1991-2001. 


\section{Figure captions}

Fig. 1 Dose-response relationships between relative growth of $S$. cerevisiae YPH499XU and IL concentration. Yeast growth activity was evaluated by $\mathrm{OD}_{660}$ at $48 \mathrm{~h}$ of the culture in the presence of IL at the subscribed concentration, and expressed as a percentage of the value without IL addition. Open circles: EmimOAc, and closed circles: ChOAc. The error bars indicate the standard deviation from three independent experiments.

Fig. 2 (A) Cellulose saccharification percentage and (B) hemicellulose saccharification percentage obtained using data at 72-h saccharification of IL-pretreated bagasse with different number of washes. The $100 \%$ cellulose and hemicellulose saccharification corresponds to $43.8 \mathrm{~g} / \mathrm{L}$ glucose and $28.5 \mathrm{~g} / \mathrm{L}$ xylose, respectively. Open bars: EmimOAc; closed bars: ChOAc. The error bars indicate the standard deviation from three independent experiments.

Fig. 3 Time courses of glucose, xylose, and ethanol concentration during fermentation of sugars saccharified from IL-pretreated bagasse with different number of post-pretreatment washes. Number of washes $=$ (A) 1, (B) 2, (C) 3, (D) 4, (E) 5, and (F) 10. Open keys: EmimOAc; closed keys: ChOAc. Circles: glucose concentration; triangles: xylose concentration; squares: ethanol concentration. The error bars indicate the standard deviation from three independent experiments. 
Fig. 4 (A) Ethanol yield on original bagasse basis. The yield was obtained using data at 48-h fermentation of sugars saccharified from IL-pretreated bagasse with different number of washes. The $100 \%$ ethanol yield corresponds to $29.0 \mathrm{~g} / \mathrm{L}$ ethanol. (B) Ethanol yield on saccharified sugar basis. The yield was obtained using data at 48-h fermentation of sugars saccharified from IL-pretreated bagasse with different number of washes. Open bars: EmimOAc; closed bars: ChOAc. The error bars indicate the standard deviation from three independent experiments. 
Table 1. Pretreatment capability and inhibitory effect of EmimOAc or ChOAc.

\begin{tabular}{|c|c|c|c|c|}
\hline \multirow{2}{*}{ IL } & \multicolumn{2}{|c|}{$\begin{array}{l}\text { Pretreatment capability of IL } \\
\text { for bagasse powder }^{\text {a }}\end{array}$} & \multicolumn{2}{|c|}{$\begin{array}{l}\text { Inhibitory effect of IL } \\
\text { on cellulase and yeast }{ }^{b}\end{array}$} \\
\hline & $\begin{array}{l}\text { Cellulose } \\
\text { saccharification } \\
\text { percentage }[\%]\end{array}$ & $\begin{array}{l}\text { Hemicellulose } \\
\text { saccharification } \\
\text { percentage }[\%]\end{array}$ & $\begin{array}{l}\mathrm{cEC}_{50} \\
{[\mathrm{wt} \%]}\end{array}$ & $\begin{array}{l}\mathrm{yEC}_{50} \\
{[\mathrm{wt} \%]}\end{array}$ \\
\hline $\begin{array}{l}\text { Control } \\
\text { without IL }\end{array}$ & $23.6 \pm 1.5$ & $9.2 \pm 0.2$ & ND & ND \\
\hline EmimOAc & $87.5 \pm 1.2$ & $67.8 \pm 0.3$ & $16.2 \pm 0.3$ & $0.6 \pm 0.02$ \\
\hline ChOAc & $92.7 \pm 3.8$ & $65.3 \pm 6.7$ & $32.3 \pm 1.3$ & ND \\
\hline
\end{tabular}

The data were expressed as mean \pm standard error from three independent experiments.

$a$ Pretreatment of bagasse powder was carried out by sonication in each IL at $25^{\circ} \mathrm{C}$ for $30 \mathrm{~min}$. The cellulose and hemicellulose saccharification percentage was obtained at $72 \mathrm{~h}$ of enzymatic reaction of pretreated bagasse with sufficient post-pretreatment wash.

$b$ The $\mathrm{cEC}_{50}$ value, determined based on cellulase activity of Ctec 2 using PASC as substrate, were from our previous report (Ninomiya et al., 2015). The $\mathrm{yEC}_{50}$ value was determined based on growth activity of yeast $S$. cerevisiae YPH499XU.

ND: Not determinable 


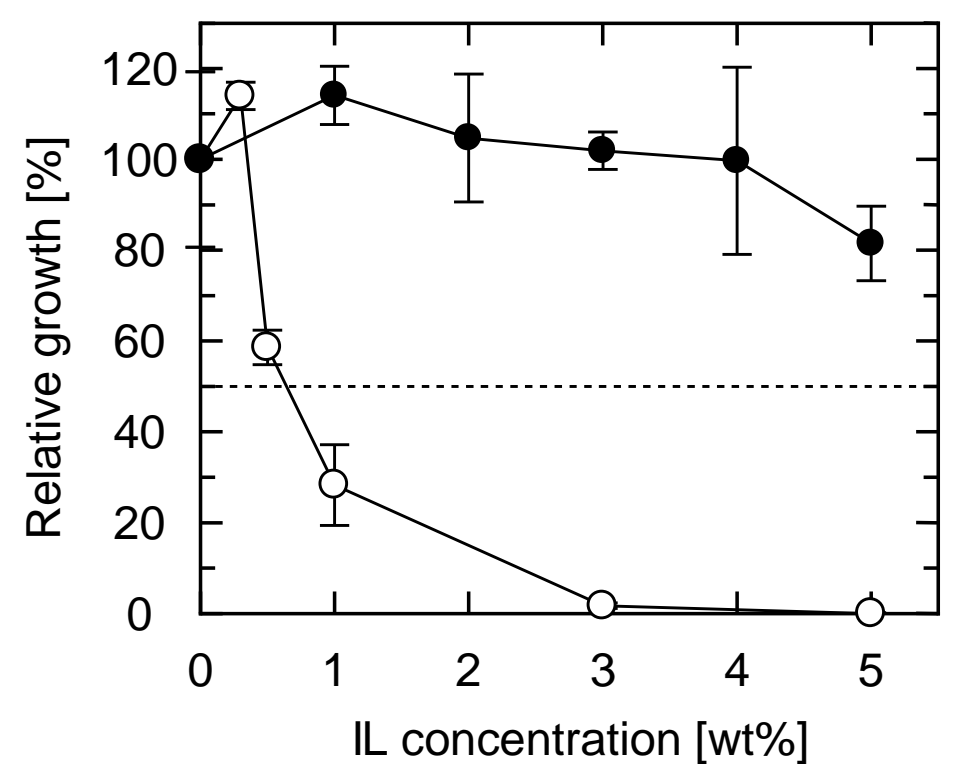

Fig. 1. Dose-response relationships between relative growth of $S$. cerevisiae YPH499XU and IL concentration. Yeast growth activity was evaluated by $\mathrm{OD}_{660}$ at $48 \mathrm{~h}$ of the culture in the presence of IL at the subscribed concentration, and expressed as a percentage of the value without IL addition. Open circles: EmimOAc, and closed circles: ChOAc. The error bars indicate the standard deviation from three independent experiments. 

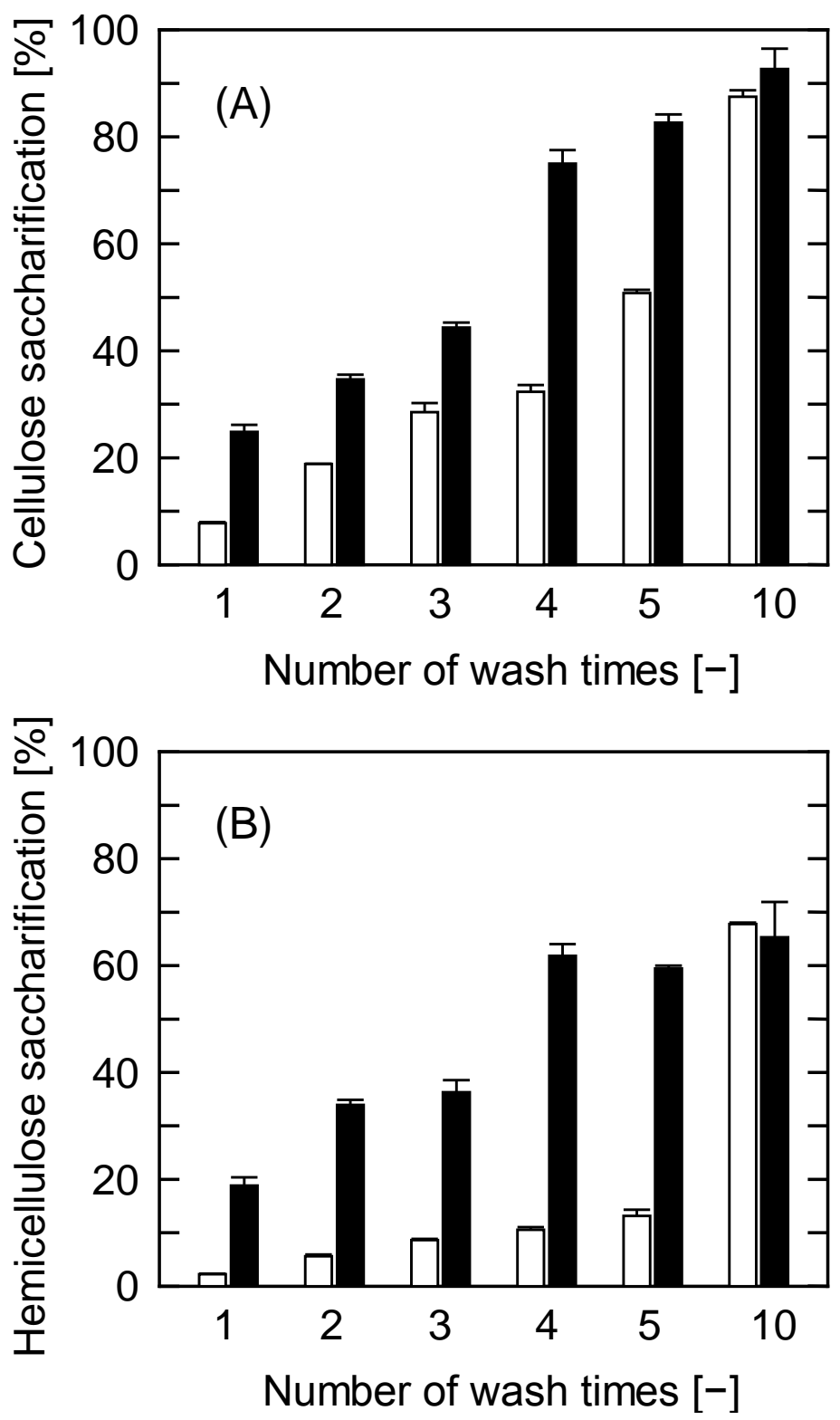

Fig. 2. (A) Cellulose saccharification percentage and (B) hemicellulose saccharification percentage obtained using data at 72-h saccharification of IL-pretreated bagasse with different number of washes. The $100 \%$ cellulose and hemicellulose saccharification corresponds to $43.8 \mathrm{~g} / \mathrm{L}$ glucose and $28.5 \mathrm{~g} / \mathrm{L}$ xylose, respectively. Open bars: EmimOAc; closed bars: ChOAc. The error bars indicate the standard deviation from three independent experiments. 

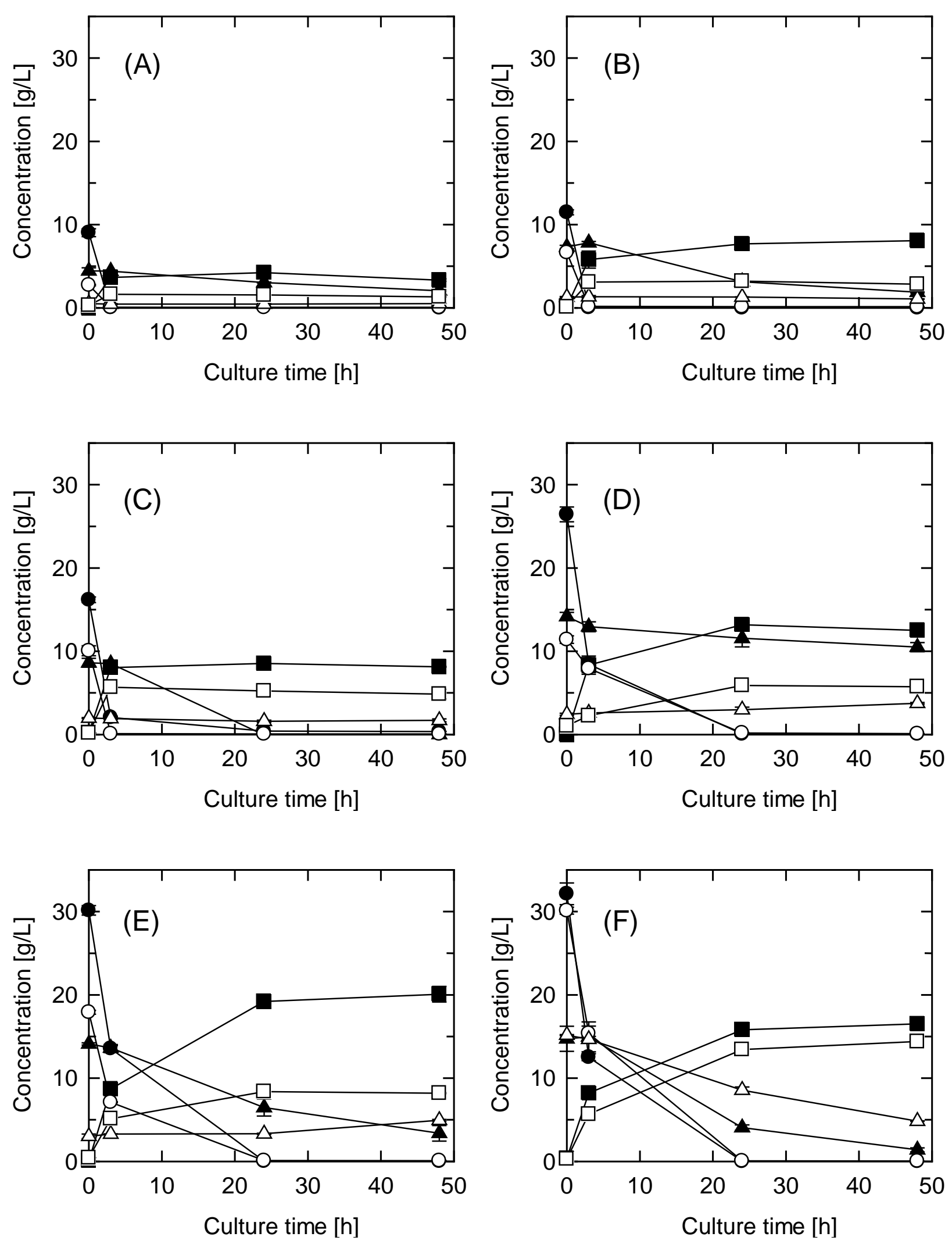

Fig. 3. Time courses of glucose, xylose, and ethanol concentration during fermentation of sugars saccharified from IL-pretreated bagasse with different number of post-pretreatment washes. Number of washes = (A) 1, (B) 2, (C) 3, (D) 4, (E) 5, and (F) 10. Open keys: EmimOAc; closed keys: ChOAc. Circles: glucose concentration; triangles: xylose concentration; squares: ethanol concentration. The error bars indicate the standard deviation from three independent experiments. 

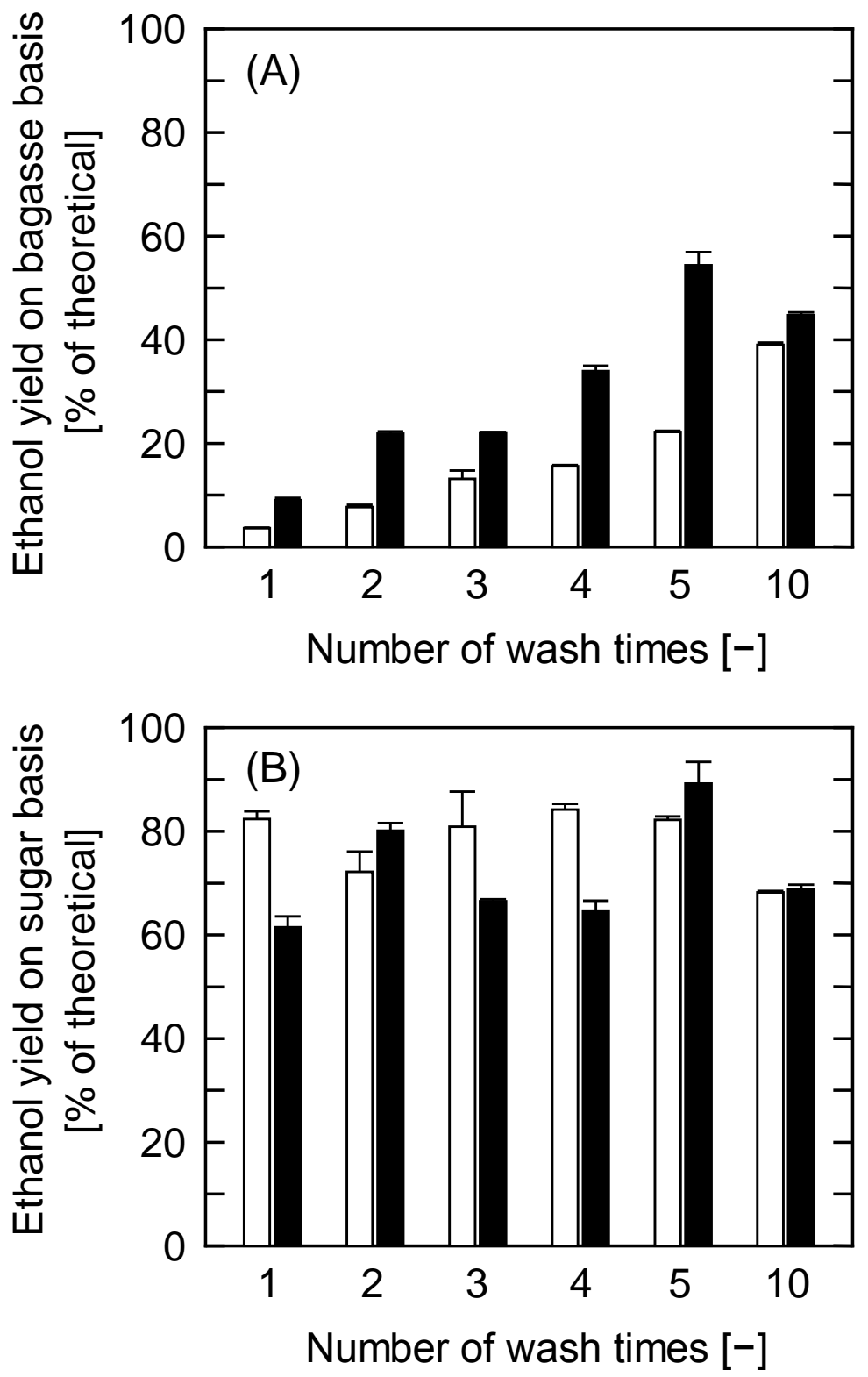

Fig. 4. (A) Ethanol yield on original bagasse basis. The yield was obtained using data at 48-h fermentation of sugars saccharified from IL-pretreated bagasse with different number of washes. The $100 \%$ ethanol yield corresponds to $29.0 \mathrm{~g} / \mathrm{L}$ ethanol. (B) Ethanol yield on saccharified sugar basis. The yield was obtained using data at 48-h fermentation of sugars saccharified from IL-pretreated bagasse with different number of washes. Open bars: EmimOAc; closed bars: ChOAc. The error bars indicate the standard deviation from three independent experiments. 